\title{
Eventos de vida estressantes entre idosos brasileiros residentes na comunidade
}

\author{
Andréa Cristina Garofe Fortes-Burgos \\ Anita Liberalesso Neri \\ Universidade Estadual de Campinas \\ Ana Paula Fabrino Bretas Cupertino \\ The University of Kansas
}

\begin{abstract}
Resumo
Foram investigados eventos de vida estressantes em 544 idosos participantes do estudo PENSA, em Minas Gerais (74,6\% de mulheres e 25,4\% de homens; média de idade $=72,11 ; D P \pm 8,29$; sendo 42,1\% de 60 a 69 anos; 39\%, de 70 a 79 e 18,9\%, 80 a 99). Os eventos compuseram as seguintes categorias: eventos relacionados à finitude (morte ou doença), problemas que afetam os descendentes, relacionados ao cuidado, eventos pontuais e bem-estar psicológico. Os homens relataram eventos pontuais (60-69), problemas dos descendentes (70-79), e finitude e bem-estar ( $\geq 80$ ); as mulheres relataram eventos pontuais (60-69), problemas dos descendentes (60-79) e finitude $(\geq 80)$. As mulheres consideraram os eventos como mais estressantes do que os homens. Resultados semelhantes têm sido relatados na literatura.
\end{abstract}

Palavras-chave: idosos; eventos de vida; estresse

\begin{abstract}
Stressful life events in Brazilian community-dwelling elderly. We examined stressful life events in 544 elderly participants of PENSA, in Minas Gerais (74.6\% women and 25.4\% men; age $=72.11$; \pm 8.29; $42.1 \%$ aged $60-69 ; 39 \% 70-79$ and $18.9 \%, 80-99)$. Stressful life events were classified into categories related to: death and illness, problems affecting children, caregiving, turning points (transitions), and psychological wellbeing. Men reported turning points (60-69), problems affecting their children (70-79), death and illness and psychological well-being ( $\geq 80$ ); women reported turning points (transitions) (60-69), problems affecting their children (60-79), and own death and illness $(\geq 80)$. Women evaluated events as more stressful than men did. There are similar results in the literature.
\end{abstract}

Keywords: elderly people; life events, stress

$\mathrm{O}$ envelhecimento acarreta numerosas e características alterações evolutivas que se manifestam em vários domínios do funcionamento biológico, psicológico e social. Parte delas traduz-se em declínio. Como o desenvolvimento é um processo finito, o envelhecimento acarreta uma diminuição na resiliência física, na capacidade de adaptação geral do organismo e na plasticidade comportamental (Baltes, 1997). Ocorrências evolutivas que implicam em perda ou ameaça de perda de controle sobre o ambiente e sobre si-mesmo têm grande potencial para serem vividas como estressantes, e, dessa forma, geralmente exercem grande demanda sobre os recursos pessoais de indivíduos de todas as idades. O declínio no funcionamento físico, o aparecimento ou o agravamento de doenças somáticas e as dificuldades com a memória são algumas das mudanças fisiológicas e psicológicas típicas da velhice, com chance de serem vividas como estressantes. Na vida social, os idosos podem ter dificuldades financeiras, resultantes da falta de trabalho, ou podem sofrer com a discriminação e o preconceito da sociedade com relação às pessoas idosas. Podem sofrer com o isolamento social e familiar, devido ao não-exercício de papéis parentais e profissionais, ou podem ter dificuldades de acesso a novas tecnologias. A perda de entes queridos ou a solidão podem acarretar problemas de ordem emocional, tais como a ansiedade e a depressão, quando os indivíduos não conseguem lidar com sentimentos de desamparo, tristeza, medo, ressentimento, dúvida e com outros estados emocionais negativos.

Os eventos de vida, entre eles o casamento, o nascimento de filhos, a aposentadoria e a perda de entes queridos, marcam momentos de transição no curso de vida, assinalam a passagem de um status evolutivo para outro, refletem a passagem do tempo individual, sinalizam o desenvolvimento normal, anunciam o alcance ou o não-alcance de metas e expectativas individuais e sociais e, quando relacionados a declínio, doenças e morte, confrontam o indivíduo com a própria finitude. Tais eventos influenciam o curso do desenvolvimento humano, bem como orientam a personalidade rumo ao enfrentamento, pois são situações que, de alguma forma, desafiam o ajustamento biológico, social e psicológico. Eventos de vida adversos, 
relacionados a perdas e a incontrolabilidade têm grande potencial para serem vividos como muito estressantes para os recursos dos idosos (Aldwin \& Gilmer, 2004; Davies, 1996; Fortes \& Neri, 2004; Neri \& Fortes, 2006).

De acordo com estudos sob os paradigmas de ciclo de vida (life cicle) e desenvolvimento ao longo do curso de vida (life span), os eventos de vida podem ser classificados como normativos e não-normativos (Baltes, 1987; Lowenthal, Thurnner, \& Chiriboga, 1975; Neugarten, 1968; Neugarten \& Hagestad, 1976). No primeiro caso, incluem-se ocorrências comuns ou esperadas para determinado grupo sócio-econômico, etário e sócio-histórico, como por exemplo, a oportunidade de alfabetização na infância, a maternidade na vida adulta e a aposentadoria da velhice. No grupo de eventos não-normativos encontram-se aqueles cuja ocorrência é incomum e inesperada e podem afetar o curso do desenvolvimento de forma peculiar. Sofrer um grave acidente ou ganhar na loteria são exemplos de eventos não-normativos do curso de vida.

Estudos que avaliaram a frequência de eventos estressantes na velhice revelaram que os idosos enfrentam mais problemas relacionados à aposentadoria, perda de entes queridos e doenças crônicas (Aldwin, Sutton, \& Lachman, 1996; Folkman, Lazarus, Pimley \& Novacek, 1987). Estudos com populações de idosos em diferentes culturas revelaram problemas relacionados à perda do cônjuge, dificuldades financeiras, saúde do cônjuge, demandas excessivas de papéis sociais e perda de um filho (Cervantes, Padilla, \& deSnyder, 1990; Murrel, Norris, \& Hutchins, 1984).

Na presente investigação foi avaliada a experiência de eventos estressantes entre idosos. Os eventos de vida estudados pertencem às seguintes classes: (a) eventos de vida normativos ou comuns ao envelhecimento (por exemplo, aposentadoria), relacionados ao desempenho de papéis normativos, que perduram no tempo e entremeiam episódios de crise e adaptação (por exemplo, cuidar de outro adulto ou idoso); e (b) eventos de vida não-normativos ao envelhecimento, vividos como crises (por exemplo, divórcio, morte de um descendente), e eventos de natureza existencial, com efeitos mais ou menos duradouros, deflagrados, ou não, por eventos de crise (por exemplo, solidão, crise espiritual, falta de significado na vida).

Os objetivos do presente estudo compreenderam: descrever a experiência de eventos estressantes entre mulheres e homens idosos pertencentes a três grupos de idade; e estabelecer uma tipologia de eventos estressantes entre idosos brasileiros residentes na comunidade.

\section{Método}

\section{Participantes}

Os idosos integravam o Estudo do Envelhecimento Saudável (PENSA $)^{1}$, para o qual foram selecionados pelo método de amostragem sistemática. Para tanto, foram visitados todos os domicílios dos bairros da cidade de Juiz de Fora, $\mathrm{MG},{ }^{2}$ cujo percentual de idosos (60 anos e mais) residentes era superior a $15 \%{ }^{3}$ (14 bairros, incluindo o Centro), segundo dados do Censo 2000 do Instituto Brasileiro de Geografia e Estatística (IBGE,
2000). Recrutadores treinados foram de porta-em-porta para identificar residências que tivessem pelo menos um morador idoso em condições físicas e cognitivas e que quisesse responder aos instrumentos da pesquisa. No total, foram visitados 7089 domicílios, dentre os quais foram identificados 1686 com pelo menos um idoso. Novecentos e cinqüenta e seis idosos aceitaram participar do estudo (56\%), 614 recusaram (36\%) e 116 (8\%) pareceram física ou cognitivamente incapacitados ${ }^{4}$. Todos os idosos que aceitaram participar do estudo preencheram uma ficha de recrutamento e assinaram um Termo de Consentimento Livre e Esclarecido. A entrevista era agendada para uma data e um horário que melhor atendiam à disponibilidade do participante.

Cada entrevista foi realizada numa única sessão, de aproximadamente duas horas, na própria residência do entrevistado, o que permitia certa flexibilidade com relação à aplicação do questionário, podendo ser interrompido quantas vezes fossem necessárias. Desta forma, atentou-se para as necessidades de cada indivíduo, visando minimizar qualquer desconforto, especialmente para os idosos mais velhos $(\geq 80$ anos). O questionário envolveu itens de auto-relato, de natureza sócio-demográfica, física, psicológica e sociológica. Os dados foram coletados entre 2002 a 2003 . As respostas foram anotadas num protocolo, conferidas pelos entrevistadores e em seguida inseridas num banco de dados, por meio do programa estatístico SPSS 12.0

Para esta investigação foram selecionados 544 dentre os 956 participantes do PENSA. O critério de seleção da amostra foi terem relatado que viveram eventos estressantes nos últimos cinco anos. Dos 544 idosos, 74,6\% eram mulheres e 25,4\% eram homens; a idade média dos participantes foi de 72,1 anos ( $d p$ = 8,29); 42,1\% tinham entre 60 e 69 anos, 39\% entre 70 e 79 anos e $18,9 \%$ tinham 80 anos e mais. Cinqüenta por cento eram casados, 39,5\% viúvos, 6,25\% solteiros, e 4,6\% divorciados, desquitados ou separados. Metade dos idosos era alfabetizada ou tinha o curso primário, outros $17 \%$ tinham o ginasial e $21 \%$ o curso colegial. Dez por cento tinham curso superior, completo ou incompleto.

\section{Materiais}

Dois instrumentos de autoria de Aldwin et al. (1996) foram traduzidos e adaptados semanticamente da língua inglesa para a língua portuguesa (Cupertino, 2001; Fortes-Burgos, Neri, \& Cupertino, 2008), para efeito do uso na pesquisa: o Inventário de Eventos Estressantes e a Avaliação da Intensidade de um Evento de Vida Negativo.

Inventário de Eventos Estressantes. Consiste em duas listas de eventos estressantes do curso de vida. A primeira lista de eventos corresponde a situações positivas, a qual não foi utilizada por este estudo. A segunda lista - utilizada nesta pesquisa - é composta por 31 itens que descrevem eventos negativos, vividos pelas pessoas idosas em diferentes pontos do curso da vida, como por exemplo, problemas de saúde, perdas de entes queridos, problemas vividos pelos descendentes, os quais influenciam o curso do desenvolvimento e do envelhecimento. Os participantes indicam quais dos eventos listados estiveram presentes em sua vida e mencionam a época em que ocorreram - se no último 
ano, nos últimos cinco anos, há mais de cinco anos, ou se não aconteceram. Para efeito das análises pretendidas por este estudo, foram consideradas somente as respostas dos eventos relatados como ocorridos nos últimos cinco anos, os quais expressam experiências próprias do processo de envelhecimento.

Avaliação da Intensidade de um Evento de Vida Negativo. Depois de responderem ao inventário de eventos de vida negativos, a pesquisa solicitava que cada participante apontasse um evento que, a seu ver, tinha sido o mais estressante de todos os que havia apontado. Então, era introduzida uma questão que solicitava avaliação pessoal do impacto desse evento estressante: "Em uma escala de 1 a 7, que nota daria para este evento em termos de ser estressante?" (sendo 1 = "nada estressante" e 7 = “muito estressante”).

\section{Resultados}

Análises univariadas e multivariadas foram realizadas buscando estabelecer tendências gerais e padrões de relações entre as variáveis. Serão apresentados os dados relativos à frequência, às categorias e à intensidade de eventos estressantes experimentados pelos idosos nos últimos cinco anos. As categorias foram criadas mediante agrupamento dos eventos listados, como forma de viabilizar as análises subsequentes, e para a qual se levou em conta a literatura especializada. Para a avaliação da intensidade do estresse, considerou-se somente o evento apontado pelos sujeitos como o mais estressante.

\section{Eventos estressantes nos últimos cinco anos}

De acordo com os objetivos já expostos, foram analisados os eventos estressantes, considerando-se apenas os ocorridos nos últimos cinco anos. Os eventos mais frequentes foram relacionados a "outra morte/perda" (30,7\%), e “problemas de saúde” do próprio idoso (12,9\%). A Tabela 1 mostra a distribuição de frequência para todos os eventos ocorridos nos último cinco anos.

A partir da análise descritiva dos eventos, foram criadas categorias, de acordo com a literatura sobre os eventos de vida e o envelhecimento humano, as quais foram utilizadas no restante da análise. As cinco categorias criadas foram as seguintes.

(1) Eventos relacionados à finitude. Referem-se à morte de pessoas amadas ou problemas de saúde da própria pessoa ou de alguém próximo, que representam ameaça à continuidade da vida. O que está em jogo aqui não é especificamente a perda de entes queridos, mas o fato de que sua morte ou seu adoecimento prenunciam a morte e a dependência do próprio idoso (inclui os itens 5, 12, 13, 21 e 23 da Tabela 1).

(2) Eventos que afetam os descendentes. São ocorrências cujo curso ou cujos efeitos não podem ser alterados pelo idoso, porque dependem de circunstâncias ligadas à vida de outrem. Segundo a literatura, dentre as ocorrências que afetam a descendência que podem afetar os idosos, as mais importantes são as que influenciam a vida dos descendentes (filhos, netos e bisnetos), em parte por causa do apego afetivo que une membros da família e que dão origem à empatia e a tentativas de interferir direta ou indiretamente, em parte porque muitas
Tabela 1

Freqüência de eventos estressantes ocorridos nos últimos cinco anos

\begin{tabular}{rlc}
\hline Item & \multicolumn{1}{c}{ Evento } & Freqüência \\
\hline 23 & Outra morte/perda & 30,7 \\
5 & Problemas saúde & 12,9 \\
16 & Morte esposo/a & 6,8 \\
12 & Problema saúde esposo/a & 6,3 \\
13 & Problema saúde filhos & 5,3 \\
28 & Problemas conjugais filhos & 5,3 \\
17 & Financeiro & 5,0 \\
21 & Morte dos pais & 4,8 \\
2 & Problema com filhos & 3,9 \\
29 & Netos & 2,9 \\
25 & Falta dos filhos & 2,8 \\
27 & Problema sucesso dos filhos & 2,6 \\
8 & Solidão & 2,2 \\
11 & Problema saúde dos pais & 1,5 \\
20 & Evento macrossocial & 1,5 \\
24 & Separação pessoas amadas & 1,5 \\
31 & Cuidando de outro adulto & 1,3 \\
6 & Problemas saúde mental & 0,9 \\
3 & Problema no trabalho & 0,6 \\
18 & Divorcio/separação & 0,6 \\
10 & Falta de significado na vida & 0,4 \\
30 & Aposentadoria & 0,4 \\
9 & Crise espiritual & 0,2 \\
\hline & &
\end{tabular}

ocorrências dizem respeito ao desempenho de papéis sociais cujo adequado cumprimento pelos descendentes confirma os esforços educativos dos pais. Destes eventos pode-se dizer que seu efeito recai também sobre o senso de integridade dos idosos, de tal sorte que não é fácil separar o que diz respeito a sentimentos para com outros significativos de sentimentos para consigo mesmos (itens 2, 24, 25, 27, 28 e 29).

(3) Eventos relacionados ao cuidado. São relacionados a pressões externas (geradas pelo curso de uma doença, pelo aumento de despesas, por conflitos familiares e pela competição do cuidado com papéis profissionais e familiares) ou internas (tristeza, ressentimento, compaixão, medo, dúvida, etc.) geradas pelo exercício do cuidado prestado a um outro adulto significativo para o idoso e que está em situação de fragilidade ou doença (itens 11 e 31 ).

(4) Eventos que afetam o bem-estar psicológico. São relacionados ao ajustamento pessoal na velhice, expressos em termos de ameaça ao senso de significado, sentimentos de solidão, problemas de saúde mental - reais ou presumidos - e crise espiritual (itens 6, 8, 9 e 10).

(5) Eventos pontuais. Entre eles estão alguns eventos típicos da velhice, tais como a aposentadoria - em muitos casos, com perdas de papéis sociais relevantes para o indivíduo, ou empobrecimento devido ao afastamento da vida produtiva - ou eventos que marcam uma perturbação no que é considerado como curso normal do desenvolvimento, tais como divórcio, alterações ocorridas no ambiente macrossocial e problemas no trabalho (itens 3, 17, 18, 20 e 30).

Os dados revelaram maior frequência de eventos relacionados à finitude - morte de entes queridos e problemas de saúde próprios e de entes queridos (66\%), seguidos de eventos que afetam a 
vida dos descendentes (19\%). Dentre os menos relatados pelos idosos estiveram os eventos pontuais $(8 \%)$, eventos que afetam o bem-estar $(4 \%)$ e aqueles relacionados ao cuidado (3\%).

Os idosos foram comparados pelos critérios de gênero e idade em relação aos diferentes tipos de eventos. Os testes estatísticos indicaram diferenças significativas: (1) de gênero; as mulheres mencionaram mais eventos de finitude e eventos que afetam a descendência, enquanto que os homens assinalaram mais eventos pontuais ou de crise (teste qui-quadrado: $\chi^{2}=11,35$; $g l=4 ; p=0,023)$; e (2) de idade; uma parte dos idosos mais jovens ( $<70$ anos) relatou mais eventos pontuais, enquanto um grupo de participantes entre 60 e 79 anos relatou mais eventos que afetam a descendência. Os idosos mais velhos ( $\geq 80$ anos) relataram com maior frequência eventos relacionados à finitude (teste qui-quadrado: $\chi^{2}=19,75 ; g l=8 ; p=0,011$ ).

A seguir foram realizadas análises comparativas levando em conta a interação entre as variáveis gênero e idade com relação à experiência de eventos estressantes. As análises permitiram concluir que houve diferença significativa com relação à experiência de eventos estressantes entre os grupos (teste exato de Fisher: $p=0,009$ ). A Tabela 2 resume a distribuição dos eventos entre os grupos.

$\begin{aligned} & \text { Tabela } 2 \\
& \text { Experiência de eventos estressantes pelos grupos de homens } e \\
& \text { mulheres, considerando as três faixas etárias selecionadas }\end{aligned}$
\begin{tabular}{cccc}
\hline Grupos & $\mathbf{6 0 - 6 9}$ anos & $\mathbf{7 0 - 7 9}$ anos & \multicolumn{1}{c}{$\mathbf{8 0}$ anos } \\
\hline Homens & Pontuais & Descendência & $\begin{array}{l}\text { Finitude / } \\
\text { Bem-estar }\end{array}$ \\
Mulheres & $\begin{array}{l}\text { Descendência / } \\
\text { Pontuais }\end{array}$ & Descendência & Finitude \\
\hline
\end{tabular}

porque morrer significa renunciar à vida neste mundo" (p.31). Segundo a autora, mesmo sendo algo natural e previsível, a morte transforma-se num terrível e inexprimível assunto a ser evitado de todas as maneiras na sociedade moderna. Talvez porque ela nos relembre nossa vulnerabilidade humana, apesar de todos os avanços tecnológicos (Kübler-Ross, 1975, p. 30).

O segundo tipo de evento mais apontado pelos idosos nesse estudo diz respeito às situações que afetam os descendentes, como por exemplo, problemas financeiros ou conjugais dos filhos ou netos. De acordo com a literatura sobre os eventos de vida no envelhecimento, tais ocorrências desafiam o senso de continuidade e integridade do self - ao significar para o idoso que ele tenha falhado como pai ou avô e como educador, responsável pela transmissão de valores e orientações necessárias para a vida de seus descendentes. Tais ocorrências podem ser sentidas pelo idoso como altamente incontroláveis.

O modelo desenvolvido por Schulz e Heckhausen (1998) sobre o controle primário e secundário indica a existência de três tipos de experiências capazes de gerar dificuldades de adaptação: experiências normativas enfrentadas quando os indivíduos têm que ampliar suas competências; declínios no processo de desenvolvimento, característicos da velhice; e eventos negativos não normativos. Pode-se dizer que tanto os eventos que afetam os descendentes quanto os eventos relacionados à finitude são marcados pela falta de controle do indivíduo. A
Foram consideradas as avaliações que os idosos fizeram dos eventos estressantes experimentados nos últimos cinco anos, levando em conta o critério de intensidade. As análises de frequência revelaram que os idosos avaliaram os eventos vividos como muito estressantes $(M=5,94$; Mediana $=7 ; D P=$ 1,49). Para a realização das análises descritivas e comparativas mediante os critérios gênero e idade, optou-se por transformar os valores da escala (que variava de 1 a 7) em três categorias de análise, respeitando os valores crescentes de intensidade da escala. Esta decisão foi tomada mediante a necessidade de viabilizar as análises subsequentes, uma vez que a distribuição de frequência na escala de 7 pontos não resultou em diferenças estatisticamente significativas. As respostas de 1 a 3 pontos foram consideradas de baixa intensidade, de 4 a 6 pontos, de intensidade moderada e de 7 pontos, de alta intensidade.

Houve diferença estatisticamente significante entre os gêneros (teste qui-quadrado: $\chi^{2}=8,91 ; g l=2 ; p=0,012$ ), ou seja, maior número de mulheres avaliou os eventos como extremamente estressante do que os homens. As comparações por critério de idade mostraram que não houve diferença estatisticamente significante entre as três faixas etárias (teste qui-quadrado: $\chi^{2}=1,10 ; g l=4 ; p=0,894$ ).

Foram realizadas as análises comparativas levando em conta a interação entre as variáveis gênero e idade com relação à avaliação de intensidade dos eventos estressantes. Os resultados revelaram não ter ocorrido diferença estatisticamente significante entre os grupos (teste qui-quadrado: $\chi^{2}=10,76 ; g l=10 ; p=$ $0,376)$.

\section{Discussão}

A literatura sobre o envelhecimento mostra que este é um processo heterogêneo, caracterizado por múltiplas trajetórias e possibilidades de crescimento e declínio, num equilíbrio dialético entre perdas e ganhos (Baltes, 1997). As perdas são mais comumente experimentadas em termos da funcionalidade física, dos relacionamentos interpessoais, dos recursos financeiros e dos papéis desempenhados durante a vida adulta. Os resultados obtidos neste estudo estão condizentes com a literatura no que diz respeito ao tipo de eventos mais frequentes entre os idosos: mais da metade da amostra relatou experiências que remetem à finitude, como problemas de saúde - deles mesmos ou de pessoas próximas - e morte de entes queridos (Aldwin, 1990, 1991; Aldwin et al, 1996; Beckett, Goldman, Weinstein, Lin, \& Chuang, 2002; Chapleski, Kaczynski, Gerbi, \& Lichtenberg, 2004; Krause, 1986; Folkman et al, 1987).

Experiências que remetem à finitude podem ser compreendidas em termos de maior dependência dos outros por circunstância de incapacidades ou problemas graves de saúde, que levam o indivíduo a tomar consciência de que seu corpo - outrora saudável, capaz, forte e competente - está sofrendo transformações, rumo ao inevitável declínio. A morte dos outros pode ser vivida como uma experiência imaginária de finitude, imaginária porque, de fato, aquele que morre não é o indivíduo, mas o outro. Em um de seus trabalhos clássicos, Kübler-Ross (1975) argumenta que "é difícil morrer e sempre será, mesmo depois de aceitarmos a morte como parte integral da vida, 
incontrolabilidade destes eventos é um fator que predispõe o indivíduo ao estresse, uma vez que pouco ou quase nada pode ser feito para resolver o problema (Diehl, 1999). A falta de controle direto sobre o ambiente faz com que mecanismos motivacionais e de competências sejam ativados no sentido de compensar as perdas experimentadas (Schulz \& Heckhausen, 1998).

No caso de eventos ligados à finitude, a falta de controle pode ser ainda maior, levando os idosos a avaliar tais situações com um grau elevado de sobrecarga. Problemas graves de saúde ou a morte de entes queridos são situações sobre as quais os indivíduos pouco têm o que fazer, objetivamente, e em geral são vividas com um alto grau de emoções negativas, sentimentos de frustração e desamparo. Nestes casos, espera-se que os indivíduos lancem mão de mecanismos de adaptação e enfrentamento que minimizem os efeitos do estresse provocado pelos acontecimentos e pela falta de controle.

Nessa amostra, as mulheres avaliaram o estresse dos eventos vividos como sendo de alta intensidade, tendência que marcou uma diferença estatisticamente significativa entre os gêneros. Alguns estudos feitos anteriormente (Aneshensel, 1992; Mirowsky \& Ross, 1995) já apontavam as mulheres como o grupo que tende a avaliar os eventos como sendo mais estressantes do que os homens. Há alguns fatores a serem considerados aqui: culturalmente, as mulheres tendem a falar mais de seus problemas do que os homens, ou seja, elas queixamse mais facilmente de enfermidades e de outros problemas em geral, o que poderia aumentar as chances desse grupo relatar maior estresse, como ocorreu na pesquisa atual. Elas podem de fato perceber os eventos como sendo mais estressantes, ou sentir os efeitos do estresse mais do que os homens, por terem menos recursos materiais, serem mais oneradas física e economicamente.

Por outro lado, pode-se especular que as mulheres dessa geração terão tido muitas chances de viver situações de incontrolabilidade associadas a preconceitos e a práticas discriminativas em relação às mulheres nos ambientes de trabalho, nos transportes, na escola e na vida em geral. Queixar-se pode ter funcionado para essas mulheres como uma estratégia de sobrevivência, uma vez que com frequência as reclamações são úteis para chamar a atenção, para ganhar controle secundário sobre o ambiente e para evitar mais punição.

Os homens relataram mais eventos pontuais, e avaliaram a intensidade do estresse provocado por tais eventos como moderada ou alta. Dentre os eventos considerados pontuais ou de crise estão a aposentadoria, o divórcio, as perdas financeiras, que são mais cruciais para os homens do que para as mulheres, pelo menos dessa geração. Alguns são típicos da velhice e outros podem marcar os demais períodos do curso da vida. É importante ressaltar que nessa coorte de idosos a maioria das mulheres foram donas-de-casa e, portanto, não tiveram nenhum tipo de trabalho formal. As situações estressantes advindas da aposentadoria foram marcadamente relatadas pelos homens, bem como as perdas financeiras decorrentes da saída destes do mercado de trabalho.

Os eventos pontuais são ocorrências normativas ou esperadas no curso da vida, cujo enfrentamento pode ser beneficiado por mecanismos de socialização antecipatória (Neugarten, 1968), como por exemplo, no caso de aposentadoria, cuja ocorrência é normalmente esperada por volta dos 60 anos, para a qual o indivíduo pode realizar um planejamento, avaliar as mudanças esperadas, buscar mais informações, e assim, estar mais preparado para enfrentar a nova condição. $\mathrm{O}$ fato de estar mais preparado para uma situação pode beneficiar o enfrentamento pessoal, aumentando o senso de controle sobre o ambiente e sobre as próprias emoções (Neugarten, 1968), o que explicaria em parte, porque os homens avaliaram o estresse advindo dos eventos relatados como tendo menor intensidade em comparação com as mulheres.

Quando considerados os critérios de gênero e idade em interação, nota-se que houve diferenças estatisticamente significantes entre os grupos. As mulheres de 60 a 79 anos relataram mais eventos que afetam os descendentes e as de 80 ou mais relataram eventos relacionados à finitude. Os homens de 60 a 69 relataram mais eventos pontuais, os de 70 a 79 anos eventos que afetam a descendência e os de 80 ou mais relataram eventos relacionados ao bem-estar psicológico. Vale ressaltar que os eventos que afetam diretamente a vida de descendentes são passíveis de influenciar o bem-estar dos idosos.

$\mathrm{O}$ presente estudo mostrou que os problemas com os descendentes podem afetar as mulheres no início da velhice e os homens na metade da velhice sendo, portanto, considerados como eventos estressantes bem presentes no envelhecimento. Houve maior variabilidade entre os homens, comparando os dois grupos etários formados entre 60 e 79 anos. Já entre as mulheres, houve predomínio de eventos que afetam a descendência nessas duas faixas etárias, sugerindo a importância atribuída pelo gênero feminino aos problemas que afetam os filhos ou os netos. Martire, Stephens e Townsend (2000) examinaram o estresse em papéis centrais - como ser mãe, esposa ou profissional - entre mulheres de meia-idade. As mulheres que tiveram problemas com os filhos relataram maiores consequências negativas associadas ao estresse, comparadas com aquelas que viveram situações estressantes associadas aos papéis de esposa ou de profissional.

Os idosos mais velhos (acima dos 80 anos) relataram com maior frequência problemas de saúde e morte de entes queridos, além de situações ligadas ao bem-estar pessoal, como sentimentos de solidão, problemas de saúde mental, reais ou presumidos, e crise espiritual. A proximidade da morte, do fim torna-se característica dos últimos anos do curso da vida. Com ela, questões espirituais podem se tornar cada vez mais frequentes e relevantes para o idoso mais velho. $\mathrm{O}$ aparecimento ou o agravamento de doenças com o passar dos anos traz a esta etapa do envelhecimento desafios adaptativos, principalmente, relacionados à manutenção da autonomia e independência, e do controle sobre o ambiente e sobre si-mesmo.

\section{Conclusão}

Os dados provenientes deste estudo mostram que situações relacionadas a perdas - especialmente aquelas que remetem à finitude - estão fortemente presentes na vida de idosos, como tem sido relatado pela literatura internacional. O reconhecimento de aspectos comuns à população idosa permite à comunidade 
científica formar um corpo consistente de conhecimentos acerca de características esperadas para o envelhecimento, em diferentes culturas. A experiência com idosos em diferentes situações (em atendimento clínico, em pesquisa, em grupos de convivência) tem revelado o maior temor dos idosos com o avançar da idade: a perda da autonomia e da independência para realizar as atividades da vida diária, como consequência de doenças incapacitantes. Por esta razão, compreende-se o fato de que eventos que remetem a um possível quadro de enfermidade, dependência e falta de controle possam representar alto nível de estresse para os idosos, particularmente, aqueles com idade acima de 80 anos, cuja realidade aproxima-se a cada dia deste quadro temido.

Por outro lado, comparações entre grupos de idosos, por gênero e faixas etárias, permitem-nos vislumbrar aquilo que tem sido amplamente divulgado pelos estudiosos da Gerontologia atual: a heterogeneidade do envelhecimento. As dificuldades e os desafios da velhice podem ser vividos de maneiras diferentes por homens e mulheres idosos. Particularmente, nesta pesquisa, podemos concluir que homens e mulheres diferem com relação às situações consideradas estressantes, bem como a maneira como avaliam o estresse advindo dos problemas enfrentados. Tais diferenças refletem aspectos particulares da coorte estudada, ou seja, dos idosos que viveram situações semelhantes de acordo com o contexto sócio-histórico em que estão inseridos. Pesquisas que avaliem futuras coortes poderão encontrar resultados diferentes destes apresentados, uma vez que mudanças no ambiente macrossocial, no desempenho de papéis - profissionais e na família - vêm mudando de forma acelerada nas últimas décadas. Futuras pesquisas poderão avaliar a experiência de eventos estressantes entre idosos residentes em diferentes localidades brasileiras, com diferentes realidades sócio-econômicas e culturais.

\section{Referências}

Aldwin, C. M. (1990). The Elders Life Inventory (ELsi): Egocentric and nonegocentric stress. In M. A. P. Stephens, S. E. Hobfoll, J. H. Crowther \& D. L. Tennenbaum (Orgs.), Stress and coping in late life families. Nova York: Hemisphere.

Aldwin, C. M. (1991). Does age affect the stress and coping process? Implications of age differences in perceived control. Journal of Gerontology, 46, 171180.

Aldwin, C. M., \& Gilmer, D. F. (2004). Health, illness, and optimal aging: biological and psychological perspectives. California: Sage.

Aldwin, C. M., Sutton, K. J., \& Lachman, M. (1996). The development of coping resources in adulthood. Journal of Personality, 64, 837-871.

Aneshensel, C. S. (1992). Social stress: Theory and research. Annual Review of Sociology, 18, 15-38.

Baltes, P. B. (1987). Theoretical propositions of life-span developmental psychology: on the dinamics between growth and decline. Developmental Psychology, 5, 611-626.

Baltes, P. B. (1997). On the incomplete architecture of human ontogeny: Selection, optimization, and compensation as foundation of development theory. American Psychologist, 52(4), 366-380.
Beckett, M., Goldman, N., Weinstein, M., Lin, I. F., \& Chuang, Y. L. (2002). Social environment, life challenge and health among the elderly in Taiwan. Social Science and Medicine, 55(2), 191-209.

Cervantes, R. C., Padilla, A. M., \& deSnyder, N. S. (1990). Reliability and validity of the Hispanic Stress Inventory. Hispanic Journal of Behavioral Sciences, 12, 79-82.

Chapleski, E. E., Kaczynski, R., Gerbi, S. A., \& Lichtenberg, P. A. (2004). American Indian elders and depression: short- and long-term effects of life events. Journal of Applied Gerontology, 23, 40-57.

Cupertino, A. P. F. B. (2001). Tradução para português e inglês. Escalas de estresse, avaliação e estratégias de enfrentamento. Juiz de Fora: Estudo PENSA.

Davies, A. D. M. (1996). Life event, health, adaptation and social support in the clinical psychology of late life. In R. T. Wood (Org.), Handbook of the Clinical Psychology of Aging. Nova York: Wiley.

Diehl, M. (1999). Self-development in adulthood and aging: The role of critical life events. In C. D. Ryff \& V. W. Marshall (Orgs.), The self and the society in aging processes (pp.150-183). Nova York: Springer.

Folkman, S., Lazarus, R. S., Pimley, S., \& Novacek, J. (1987). Age Differences in stress and coping processes. Psychology and Aging, 2, 171-184.

Fortes, A. C. G., \& Neri, A. L. (2004). Eventos de Vida e Envelhecimento Humano. In A. L., Neri \& M. S. Yassuda (Orgs.), Velhice bem-sucedida. Campinas, SP: Papirus.

Fortes-Burgos, A. C. G., Neri, A. L., \& Cupertino, A. P. F. B. (2008). Eventos estressantes, estratégias de enfrentamento, auto-eficácia e sintomas depressivos entre idosos residentes na comunidade. Psicologia Reflexão $e$ Crítica, 21(1), 74-82.

Instituto Brasileiro de Geografia e Estatísticas (2000). Censo Demográfico. Rio de Janeiro: Autor.

Krause, N. (1986). Stress and sex differences in depressive symptoms among older adults. Journal of Gerontology, 6, 727-731.

Kübler-Ross, E. (1975). Morte: Estágio final da evolução (A. M. Coelho, Trad.). Rio de Janeiro: Record.

Lowenthal, M. F., Thurnner, M., \& Chiriboga, D. A. (1975). Four stages on life. San Francisco: Josey-Bass.

Martire, L. M., Stephens, M. A. P., \& Townsend, A. L. (2000). Centrality of women's multiple roles: beneficial and detrimental consequences for psychological well-being. Psychology and Aging, 15(1), 148-156.

Mirowsky, J., \& Ross, C. E. (1995). Sex differences in distress: Real or artifact? American Sociological Review, 60, 449-468.

Murrel, S., Norris, F. H., \& Hutchins, G. L. (1984). Distribution and desirability of life events in older adults: Population and policy implications. Journal of Community Psychology, 12, 301-311.

Neri, A. L., \& Fortes, A. C. G. (2006). A dinâmica do estresse e enfrentamento na velhice e sua expressão no prestar cuidados a idosos no contexto da família. In E. V. Freitas, L. Py, F. A. X. Cançado, J. Doll \& M. L. Gorzoni (Orgs.), Tratado de Geriatria e Gerontologia (pp. 1.277-1.288). Rio de Janeiro: Guanabara Koogan.

Neugarten, B. L. (1968). Adult personality: toward a psychology of the life cycle. In B. L. Neugarten (Org.), Middle age and aging: a reader in social psychology (pp. 137-147). Chicago: University of Chicago Press.

Neugarten, B. L., \& Hagestad, G. D. (1976). Age and the life course. In R. H. Binstock \& E. Shanas (Orgs.), Handbook of aging and the social sciences (pp. 35-55). Nova York: Van Nostraud-Reinhold.

Schulz, R., \& Heckhausen, J. (1998). Emotion and control: A life-span perspective. In Annual Review of Gerontology and Geriatrics, vol. 17 - Focus on Emotion and Adult Development. Philadelphia, Pennsylvania: Springer. 
1. Inicialmente financiado pelo CNPq, Programa de Fixação de Doutores - PROFIX, e aprovado pelo comitê de ética em pesquisa da Universidade Federal de Juiz de Fora. Amostra total de 956 idosos. Dados coletados entre os anos de 2002 e 2003.

2. A cidade de Juiz de Fora tem aproximadamente 436.432 habitantes, sendo 10,6\% idosos (acima de 60 anos). Este índice é superior à população idosa do estado de Minas Gerais $(9,8 \%)$ e do Brasil $(8,6 \%)$.

3. O critério utilizado para selecionar bairros com uma concentração de $15 \%$ ou mais de idosos foi de otimização de custos para a pesquisa, conforme orientação da agência de pesquisa patrocinadora. O projeto foi aprovado para utilizar um ponto de corte de $20 \%$ ou mais de idosos, porém optou-se por um ponto de corte mais próximo à realidade do município.

4. Pessoas que não poderiam responder ao questionário devido a problemas como incapacidades físicas ou doenças que impediam o idoso de permanecer sentado pelo tempo da entrevista, ou problemas cognitivos, decorrentes de derrame ou outras enfermidades, que prejudicariam a legitimidade das respostas a serem dadas pelo idoso. Tais aspectos da condição do idoso foram verificados durante a visita de recrutamento, através de perguntas feitas ao próprio indivíduo ou a algum familiar sobre a capacidade do idoso para responder (sem ajuda de outros) ao questionário.

Andréa Cristina Garofe Fortes-Burgos, mestre em Gerontologia pela Universidade Estadual de Campinas, é doutoranda em Educação na mesma universidade (com bolsa de estudos da FAPESP). Endereço para correspondência: Av. Ipê Roxo, 815; Condomínio Tulipas, casa 10 (Parque Villa Flores); Sumaré, SP; CEP: 13175-658. Telefone: (19) 3306-8315.E-mail: andreafortes@gmail.com

Anita Liberalesso Neri, doutora livre-docente em Desenvolvimento Humano pela Universidade Estadual de Campinas, é professora titular na Faculdade de Educação da mesma universidade. E-mail: anitalbn@ uol.com.br

Ana Paula Fabrino Bretas Cupertino, PhD em Medicina Preventiva e Saúde Pública pela University of California, em Davis, é assistant professor na University of Kansas Medical Center. E-mail: acupertino@ kumc.edu 